\title{
Review of: "Evaluation of mRNA-1273 SARS-CoV-2 Vaccine in Adolescents"
}

daphne sawlwin

Potential competing interests: The author(s) declared that no potential competing interests exist.

The trial design, both safety and immunogenicity results were well explained. As the authors acknowledged the number of covid-19 cases was insufficient to conclude vaccine efficacy however the result from a small number of cases was nonetheless encouraging. The authors noted that $6 \%$ of (Table 1 ) of subjects in the safety population were seropositive at baseline. It would be helpful for the reviewers to understand if there were any differences in terms of frequencies of solicited systemic reactions during 7 days following the first and second vaccination, in particular, fever in seropositive and seronegative populations. Similarly, a comment relating to the reactogenicity profile by sex (male/female) subgroup would be informative. One last comment is regarding lack of information around drug-induce liver injury (which concomitant drugs or vaccine were involved etc.,) and the nature of adverse events leading to withdrawal from the study. 